\title{
ENOVELAMENTO DO FIO GUIA EM CATETER CENTRAL DE INSERÇÃO PERIFÉRICA (PICC): RARA COMPLICAÇÃO
}

\author{
Knoted guidewire in peripherally inserted central catheters (PICC): a rare complication
} Enredado del alambre guía en el catéter central de la inserción periférica (PICC): complicación infrecuente

Sirlene Cristina de Almeida Santos', Ana Paula Rolim', Nicole Acácia Cabral Nunes², Elena Bohomol ${ }^{3}$, Manoel Carlos Prieto Velhote ${ }^{4}$

RESUMO: Objetivo: Relatar a experiência vivenciada por uma equipe multiprofissional referente ao gerenciamento de um evento adverso após a passagem de cateter central de inserção periférica (PICC) por retenção do fio guia metálico. Método: Relato de experiência ocorrida em hospital geral no município de Guarulhos, São Paulo, em novembro de 2015. Resultado: Descreve-se o caso de lactente de um ano de idade, hemofílico e neuropata, que, após a passagem de PICC 3F no antebraço sem incidentes, teve seu fio guia retido, impossibilitando sua retirada. A radioscopia revelou enovelamento do fio guia, com necessidade de remoção cirúrgica. Conclusão: O procedimento cirúrgico foi realizado com sucesso. Não houve perda sanguínea de monta no campo operatório. A criança se recuperou bem, sem consequências detectáveis pela intercorrência. Notificou-se o fabricante sobre o evento ocorrido. Este caso serviu como um aprendizado para a equipe multiprofissional. Palavras-chave: Cateteres. Complicações. Procedimentos cirúrgicos operatórios. Equipe de assistência ao paciente.

ABSTRACT: Objective: To report the experience of a multiprofessional team on the management of an adverse event after the insertion of a peripherally inserted central catheter (PICC) because of the retention of the metallic guidewire. Method: An experience report concerning an occurrence at a general hospital in the city of Guarulhos, São Paulo, Brazil, in November 2015. Results: Description of the case of a 1-year-old infant, with hemophilia and neuropathy, who had its guidewire held after the insertion of a PICC 3F in the forearm without incidents, which made its removal impossible. Radioscopy revealed that the guidewire was curled up and had to be surgically removed. Conclusion: The surgical procedure was successful. There was no blood loss in the operating field. The infant recovered well, without any consequences detected because of the complication. The manufacturer was notified of the event. This case served as a learning experience for the multiprofessional team.

Keywords: Catheters. Complications. Surgical procedures, operative. Patient care team.

RESUMEN: Objetivo: Reportar la experiencia pasada por un equipo multiprofesional en referencia al manejo de un evento adverso luego del paso del catéter central de la inserción periférica (PICC) por retención del alambre metálico. Método: Relato de una experiencia sucedida en un hospital general del municipio de Guarulhos (San Pablo, Brasil), en noviembre de 2015. Resultado: Se describe el caso de un lactante de un año, hemofílico y portador de neuropatía, lo cual, luego del paso de un PICC 3F por su antebrazo sin incidencias, sufrió una retención del alambre, que imposibilitaba retirarlo. La radioscopia mostró un enredado del alambre guía, que debió retirarse por vía quirúrgica. Conclusión: El procedimiento quirúrgico se llevó a cabo con éxito. No hubo pérdida sanguínea relevante en el acto quirúrgico. El niño se recuperó bien, sin consecuencias detectables que fueron motivadas por el evento. Se notificó al fabricante sobre el inconveniente acaecido. Este caso sirve como un aprendizaje para el equipo multiprofesional.

Palabras clave: Catéteres. Complicaciones. Procedimientos quirúrgicos operativos. Grupo de atención al paciente.

Enfermeira da Unidade de Terapia Intensiva Neonatal do Hospital Carlos Chagas. E-mail: sirlene.a@hcc.com.br

${ }^{2}$ Médica Pediatra da Unidade de Terapia Intensiva Neonatal do Hospital Carlos Chagas. E-mail: nnunes.direito@gmail.com

${ }^{3}$ Enfermeira. Livre-docente da Escola Paulista de Enfermagem da Universidade Federal de São Paulo. E-mail: ebohomol@uol.com.br

«Médico Cirurgião Pediatra. Livre-docente do Instituto da Criança do Hospital das Clínicas da Faculdade de Medicina da Universidade de São Paulo. E-mail: mvelhote@uol.com.br.

Rua São Firmo, 81. Vila Ida, CEP: 05454-060. São Paulo (SP), Brasil.

Recebido: 01 abr. 2016 - Aprovado: 11 maio 2016

DOI: $10.5327 / Z 1414-4425201600020008$ 


\section{INTRODUÇÃO}

O acesso venoso durável em crianças teve um grande progresso com a instituição da passagem de cateteres por punção percutânea, pela técnica de Seldinger, em veias calibrosas no pescoço, raiz da coxa ou região subclávia, ao invés da dissecção tradicional.

O estado da arte foi atingido com o desenvolvimento de cateteres bastante finos, passíveis de serem inseridos em qualquer veia periférica, o peripherally inserted central catheter (PICC), comumente conhecido como cateter central de inserção periférica. Esses cateteres podem permanecer por longo tempo, sendo de fácil manutenção. Estão associados à baixa incidência de infecções da corrente sanguínea, desde que passados sob rígido protocolo de inserção ${ }^{1}$.

A utilização de PICC tem sido recomendada em pacientes que requerem tempo prolongado de terapia intravascular, como administração de medicamentos e quimioterápicos, transfusões sanguíneas, nutrição parenteral, além de possibilitar o monitoramento hemodinâmico ${ }^{2}$.

Normalmente os PICCs são inseridos à beira do leito por enfermeiros e médicos capacitados e habilitados para realizar o procedimento. Embora seja um procedimento seguro, estudos demonstram que complicações podem ocorrer, sejam elas relacionadas à passagem, permanência ou retirada do cateter. Estão descritas: sepse, obstrução, remoção acidental, infiltração, edema, flebite, derrame pleural, derrame pericárdico, fratura do cateter, migração do cateter, trombose, vazamento, mau posicionamento da ponta do cateter, dificuldade de remoção e embolia ${ }^{1,3-5}$. Todavia, não foi localizada na literatura a descrição de travamento do fio guia na sua retirada após a passagem desse tipo de cateter.

\section{OBJETIVO}

Relatar a experiência vivenciada por uma equipe multiprofissional no gerenciamento de um evento adverso, após a passagem de PICC, por retenção do fio guia metálico.

\section{MÉTODO}

Trata-se de um relato de experiência vivenciada por enfermeiros e médicos, por ocasião da passagem de PICC em hospital geral acreditado, no município de Guarulhos, São Paulo, ocorrida em novembro de 2015. Descreve-se um caso raro de complicação de PICC passado na veia basílica em um lactente de onde, após o acesso sem incidentes, o fio guia não saiu, ficando preso, retorcido sobre si mesmo, necessitando de intervenção cirúrgica para sua extração.

\section{RESULTADOS}

Menor, do sexo masculino, um ano de idade, internado há cinco meses na Unidade de Terapia Intensiva (UTI) Neonatal e Pediátrica, com diagnóstico de hemofilia e sequela neurológica por sangramento intracraniano, teve necessidade de um novo acesso central para infusão de Fator VIII, três vezes por semana, além de manutenção parenteral e antibioticoterapia.

A enfermeira habilitada para realizar o procedimento optou, segundo protocolo habitual do hospital, pelo PICC 3F valvulado utilizando a técnica de Seldinger, devido a suas reconhecidas vantagens, como o acesso de longa permanência ${ }^{6}$.

Após cuidados de assepsia e paramentação cirúrgica, foi realizada a punção em veia basílica esquerda com ótimo refluxo sanguíneo. Durante a introdução do cateter não houve nenhuma resistência, permitindo, assim, avançar de maneira satisfatória até atingir uma posição central, confirmada pela radioscopia.

Ao se realizar a manobra da retirada do fio guia, o mesmo apresentou resistência, não sendo possível sua retirada. Foram realizadas manobras de mudança de posicionamento do membro tracionando alguns centímetros o fio guia, porém não se obtendo sua saída. Foi solicitado auxílio do médico intensivista, que realizou várias tentativas de tracionar o fio guia, também sem sucesso.

À radioscopia, foi visualizado um enovelamento do cateter, impossibilitando sua saída e a do fio (Figura 1).

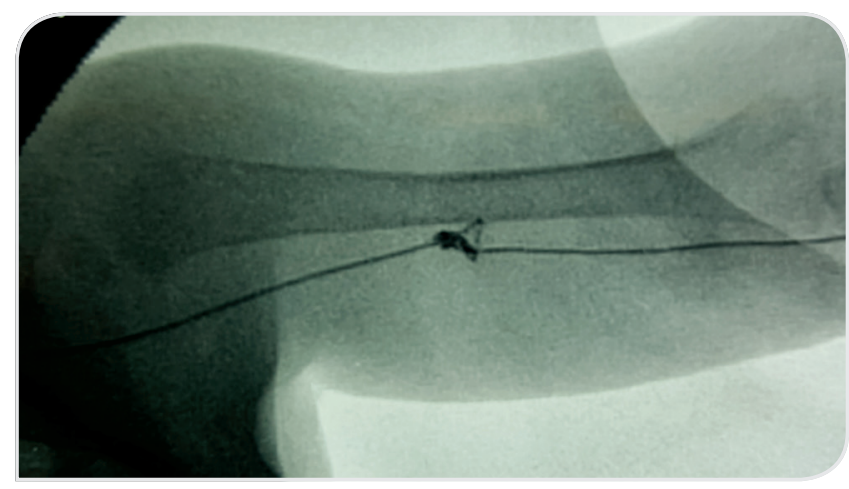

Figura 1. Radiografia do enovelamento do cateter no terço médio do braço. 
O cirurgião pediatra foi acionado para a retirada do cateter por acesso cirúrgico.

O cirurgião pediatra localizou, de forma aproximada, a posição do enovelamento do cateter, sugerido pela radiografia, na altura do terço médio do braço esquerdo. Sob anestesia local, realizou-se uma incisão longitudinal de cerca de três centímetros na face medial do braço, sendo aberta a aponeurose braquial, dissecado e reparado o nervo mediano e a artéria umeral, passando-se em seguida a localizar a veia umeral com o cateter bloqueado. Essa identificação foi facilitada pela secção da parte exteriorizada do cateter junto à pele e sua mobilização (Figura 2).

Identificada a veia umeral com o cateter no seu interior, a mesma foi seccionada, e o cateter extraído por tração não regrada. O cateter apresentava-se emaranhado, retorcido sobre si mesmo (Figura 3).

Utilizou-se o mesmo coto venoso para passagem de novo cateter, sem intercorrências, com a ponta em posição central. Foram liberadas as estruturas neurovasculares reparadas, e fechada a incisão. Apesar do paciente ser hemofílico, não houve perda sanguínea de monta no campo. A criança se recuperou bem, sem consequências detectáveis pela intercorrência. Notificou-se o fabricante sobre o evento ocorrido.

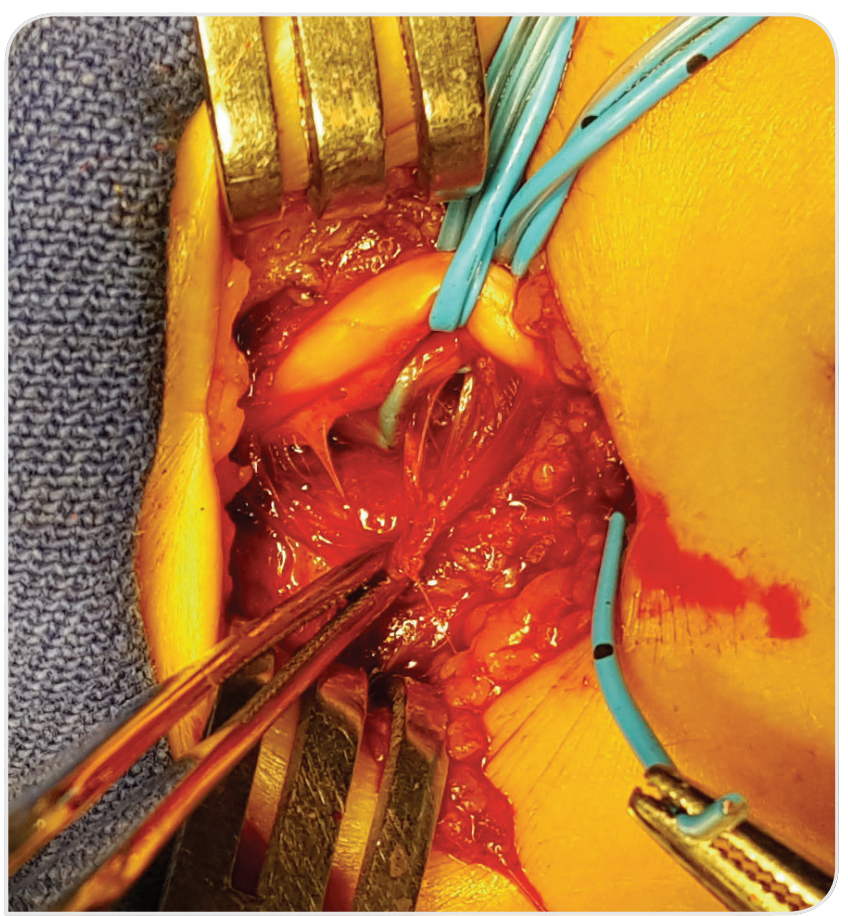

Figura 2. Dissecção da veia umeral. Nervo mediano e artéria umeral reparados com vessel loop. Cateter central de inserção periférica preso no hemostático.

\section{DISCUSSÃO}

Estão descritos na literatura vários casos de retenção do fio guia na passagem de cateteres não PICC em veias mais calibrosas. Tais veias tem espaço potencial para o cateter enovelar-se, provocando nós tanto em artérias utilizadas para hemodiálise $^{7}$ como em veias ${ }^{8,9}$.

Em busca no PubMed, não se verificou nenhum caso de fio guia metálico retido na passagem de PICC embora estudo $^{10}$ realizado em 19 UTIs no Japão relate problemas com 975 casos relacionados ao PICC, sendo um por "dificuldade de retirada do cateter". Esse estudo não descreveu o problema em minúcias, nem sua relação com o fio guia. Outro estudo ${ }^{1}$, realizado em 2.574 PICCs passados em 1.807 crianças, relatou complicações necessitando de retirada do cateter em $20,8 \%$ (11,6 complicações por mil cateteres / dia), porém sem nenhum caso semelhante ao descrito no estudo aqui apresentado.

Em revisão bibliográfica para averiguar as complicações em PICC não há menção a respeito de retenção de fio guia por ocasião da passagem do catéter ${ }^{5}$. Um estudo ${ }^{11}$ relata um nó formado no cateter de PICC passado no período neonatal, nada mencionando quanto ao fio guia metálico.

Dessa forma, sugere-se como explicação para a ocorrência do evento relatado o deslizamento inadequado do fio metálico na hora da retirada da guia metálica, que ficou preso no cateter, deformando-o e desenluvando-o. As subsequentes tentativas de retirada, avançando e tracionando o fio guia, provavelmente provocaram dobras permanentes no metal, tornando impossível sua retirada sem trauma maior para o vaso. Felizmente, o aprisionamento do fio guia ocorreu no braço, que é de fácil acesso à exploração cirúrgica, a qual

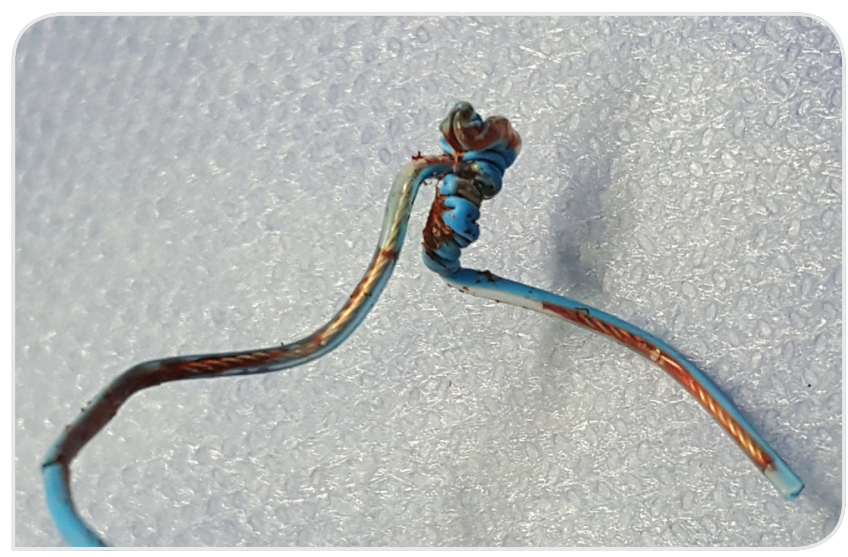

Figura 3. Imagem do fio guia extraido. 
foi realizada com facilidade e sem maiores traumas para as estruturas arteriais e nervosas ali presentes.

Após o término do procedimento, os cuidados necessários ao paciente foram prescritos e realizados. Além disso, a equipe assistencial preparou um relatório do ocorrido, solicitando à administração da instituição que tomasse providências junto ao fabricante para que esse instituísse um controle de qualidade dos cateteres disponibilizados para as instituições.

O presente relato demonstra a utilização do PICC como uma prática de assistência avançada, especializada e de alta complexidade, todavia com riscos de eventuais complicações ${ }^{12}$.

\section{CONCLUSÃO}

Foi apresentada uma complicação incomum em cateter central de inserção periférica — o enovelamento do fio guia na passagem do cateter —, necessitando de intervenção cirúrgica para sua extração.

Verifica-se também o comprometimento da equipe composta por enfermeiros, médicos intensivistas e cirurgião pediátrico, técnicos de raio- $\mathrm{X}$ e administradores, congregando esforços para resolver uma intercorrência não esperada e diminuir eventuais consequências ao paciente.

\section{REFERÊNCIAS}

1. Wrightson DD. Peripherally inserted central catheter complications in neonates with upper versus lower extremity insertion sites. Adv Neonatal Care. 2013;13(3):198-204.

2. Baggio MA, Bazzi FCS, Bilibio CAC. Cateter central de inserção periférica: descrição da utilização em UTI neonatal e pediátrica. Rev Gaúcha Enferm. 2010;31(1):70-6.

3. Jumani K, Advani S, Reich NG, Gosey L, Milstone AM. Risk factors for peripherally inserted central venous catheter complications in children. JAMA Pediatr. 2013;167(5):429-35.

4. Pettit J. Assessment of infants with peripherally inserted central catheters: Part 1. Detecting the most frequently occurring complications. Adv Neonatal Care. 2002;2(6):304-15.

5. Secoli SR, Jesus VC. Complicações acerca do cateter venoso central de inserção periférica (PICC). Ciênc Cuid Saúde. 2007;6(2):252-60.

6. Conselho Regional de Enfermagem de São Paulo. Parecer Coren-SP CAT $n^{\circ} 006 / 2010$. Técnica de Seldinger modificada para punção arterial. [acesso em 29 janeiro 2016]. Disponível em: http://portal. coren-sp.gov.br/sites/default/files/parecer_coren_sp_2010_6.pdf
7. Altıntaş G, Diken Ai, Ulaş MM, Çiçek ÖF, Özen A, Ökten S, et al. Surgical removal of a knotted and entrapped subclavian hemodialysis catheter guidewire. Ann Vasc Surg. 2012;26(3):421.e17-9.

8. Khan KZ, Graham D, Ermenyi A, Pillay WR. Case report: managing a knotted Seldinger wire in the subclavian vein during central venous cannulation. Can J Anaesth. 2007;54(5):375-9.

9. Fiala A, Glodny B, Lorenz IH. Knotted Seldinger wire. Anaesthesist. 2016;65(1):42-5.

10. Ohki Y, Maruyama K, Harigaya A, Kohno M, Arakawa H. Complications of peripherally inserted central venous catheter in Japanese neonatal intensive care units. Pediatr Int. 2013;55(2):185-9.

11. Kurtom W, Degnan A, Quast D. Knot formation of a neonatal peripherally inserted central catheter: a rare complication of placement. J Vasc Access. 2016;17(1):e15-6.

12. Oliveira CR, Neve ET, Rodrigues EC, Zamberlan KC, Silveira A. Cateter central de inserção periférica em pediatria e neonatologia: possibilidades de sistematização em hospital universitário. Esc Anna Nery. 2014;18(3):379-85. 